\title{
Intraventricular hemorrhage after ventriculoperitoneal shunt revision: a retrospective review
}

\author{
Mark Calayag, MD, Alexandra R. Paul, MD, and Matthew A. Adamo, MD \\ Department of Neurosurgery, Albany Medical Center, Albany, New York
}

OBJECT The authors review their ventriculoperitoneal (VP) shunt revisions over a 3-year period to determine the rate of intraventricular hemorrhage (IVH) and subsequent need for re-revision.

METHODS Review of medical records identified 35 pediatric patients who underwent 52 VP shunt revisions between 2009 and 2012. The presence and amount of IVH as determined by CT and the time to re-revision were documented. The reason for shunting, catheter position, and time between initial VP shunt placement and subsequent revisions were also recorded.

RESULTS After 13 (25\%) of the 52 revisions, IVH was evident on postoperative CT scans. The majority of patients had a trace amount of IVH, with only $2 \%$ having IVH greater than $5 \mathrm{ml}$. After $2(15 \%)$ of the 13 revisions associated with IVH, re-revision was required within 1 month. In contrast, the re-revision rate in patients without IVH was $18 \%$. All of the patients who developed IVH had occipital catheters.

CONCLUSIONS Some degree of IVH can be expected after approximately one-quarter of all VP shunt revision procedures in pediatric patients, but the rate of significant IVH is low. Furthermore, the presence of IVH does not necessitate an early shunt revision.

http://thejns.org/doi/abs/10.3171/2014.11.PEDS14246

KEY WORDS intraventricular hemorrhage; ventriculoperitoneal shunt; shunt revision; hydrocephalus

$\mathrm{T}$ HE revision of ventriculoperitoneal (VP) shunts for shunt failure is a fairly common neurosurgical procedure, especially in the pediatric population. Intraventricular hemorrhage (IVH) after the removal of a ventricular catheter is a known complication of this operation. Brownlee et al. ${ }^{3}$ examined this and were the first to determine a rate of IVH for catheter revisions. However, since their paper, there has been no new literature describing the hemorrhage rate, and there has not been any publication describing a revision rate after IVH. Here we retrospectively review our proximal VP shunt revisions to determine our rate of IVH as well as the rate of needing a further revision.

\section{Methods}

Approval was obtained from the Albany Medical College Institutional Review Board. The medical records of our pediatric patients who underwent revision of their VP shunt from 2009 to 2012 were reviewed. We included patients who: 1) had a revision of their proximal catheter and 2) had postoperative imaging within 3 days. Characteristics such as the etiology of hydrocephalus, time since the previous revision, presence of slit or normal ventricles, and catheter trajectory were documented. The difficulty of removing the catheter (as reported by the senior author in the operative note, with difficulty defined as the need for more 
force than usual to remove a ventricular catheter), IVH on imaging, and revision within 1 month (which we defined as an early revision) after removal were all recorded. The volume of the IVH was determined using the $\mathrm{ABC} / 2$ method. ${ }^{7}$ IVH greater than $5 \mathrm{ml}$ was considered a significant hemorrhage. The Student t-test and Fisher exact test were performed for statistical analysis of our data.

Our approach to evaluating the shunt system was standard in all patients included in the study. Patients who had radiographic and/or clinical signs of shunt failure were taken to the OR for exploration of their shunt system. Intraoperative evaluation of the shunt was performed. This included an initial evaluation of the valve and proximal catheter. If there was no flow or very little flow from the proximal catheter once it was disconnected from the valve, it was replaced through the original bur hole. A NeuroPEN (Medtronic) was routinely used to traverse the same tract. If there was evidence of bleeding within the ventricle upon replacement of the proximal catheter (i.e., direct visualization or bloody CSF), then the ventricle was irrigated through the new proximal catheter until the CSF cleared. The valve and distal catheter were then evaluated by attaching a manometer and assessing flow by monitoring the fluid column. If there was concern for distal failure, the valve was then removed and the distal catheter was evaluated separately. The valve and distal catheter were then replaced if necessary.

\section{Results}

We identified 35 patients who underwent a total of 52 VP shunt revisions. Their baseline demographic characteristics are shown in Table 1. Blood within the ventricle was demonstrated in 25\% (13 of 52) of our proximal catheter revisions; however, only 1 patient had an IVH that was greater than $5 \mathrm{ml}(2 \%)$. The patient's age, sex, or reason for VP shunting was not found to be significant in causing IVH. Additional revision (re-revision) was required within 1 month in $2(15 \%)$ of the 13 revision cases in which IVH

\section{TABLE 1. Demographic characteristics of the patients in our} series

\begin{tabular}{lc}
\hline \multicolumn{1}{c}{ Characteristic } & Value \\
\hline Average age (yrs) & 7.52 \\
\hline$\%$ male & $60.8 \%$ \\
\hline Cause of hydrocephalus & \\
\hline Congenital & $17.7 \%$ \\
\hline Chiari malformation & $13.7 \%$ \\
\hline Myelomeningocele & $11.8 \%$ \\
\hline GMH & $36.5 \%$ \\
\hline TBI & $3.9 \%$ \\
\hline Midline cyst & $9.8 \%$ \\
\hline Aqueductal stenosis & $7.8 \%$ \\
\hline Neonatal meningitis & $3.1 \%$ \\
\hline Infection & $2 \%$ \\
\hline Arachnoid cyst & $9.8 \%$ \\
\hline Posterior fossa mass & $3.9 \%$ \\
\hline
\end{tabular}

$\mathrm{GMH}$ = germinal matrix hemorrhage; $\mathrm{TBI}$ = traumatic brain injury. was identified on postoperative imaging compared with $7(18 \%)$ of the 39 cases in which postoperative imaging did not show IVH (Table 2). Furthermore, the average length of time until subsequent revision was longer after revision surgery with a postoperative finding of IVH than after revision surgery without associated IVH (219 days vs 47 days). Two (40\%) of 5 shunt revision procedures performed in patients with slit/normal ventricles were associated with subsequent IVH compared with $11(23 \%)$ of 47 procedures in patients without slit/normal ventricles, but this difference was not statistically significant $(\mathrm{p}=$ 0.58 ). Revision of occipital shunts had a $36 \%$ rate of IVH (13 of 36 procedures), while there was no postoperative IVH associated with revision of frontal VP shunts $(0$ of 16 cases), a difference that was found to be significant ( $p$ $=0.001$ ). Altogether there were 13 proximal revisions that were considered difficult. Eight (62\%) of these revisions resulted in postoperative IVH, a rate that was found to be significant $(\mathrm{p}<0.01)$. Only 1 patient from that group required an early revision.

\section{Discussion}

The association between removal of a VP shunt catheter and the subsequent development of IVH is well known and documented. ${ }^{2}$ Proposed mechanisms include growth of the choroid plexus surrounding the catheter with infiltration into holes at the catheter tip., ${ }^{1,5}$ This has been supported by histological studies demonstrating either choroid plexus or ependymal tissue obstructing the tip of the catheter. ${ }^{4}$ More recently, with the advent of neuroendoscopy, in vivo visualization of shunt tubes encased in ependymal growth and neovascularization has been described in the literature. ${ }^{11}$ Disruption of the choroid plexus upon removal of the catheter would then lead to bleeding of this highly vascular structure into the ventricular space.

Brownlee et al. ${ }^{3}$ have been the only ones to look at the rate of occurrence of IVH after VP shunt revision surgery

TABLE 2. Analysis of outcomes after proximal shunt revisions with and without postoperative IVH

\begin{tabular}{lclc}
\hline \multicolumn{1}{c}{ Variable } & No IVH & IVH & p Value* \\
\hline No. of revisions $(\mathrm{n}=52)$ & $39(75 \%)$ & $13(25 \%)$ & \\
\hline Average patient age $(\mathrm{yrs})$ & 7.2 & 10.4 & 0.17 \\
\hline$\%$ male & 54 & 62 & 0.65 \\
\hline Reason for VP shunt & & & 0.56 \\
\hline$\quad$ Congenital & 21 & 6 & \\
\hline$\quad$ TBI/GMH/tumor/infection & 18 & 7 & \\
\hline Average time before revision (mos) & 38 & 56 & 0.13 \\
\hline Normal/slit ventricles & 2 & 3 & 0.58 \\
\hline Location of VP shunt & & & 0.001 \\
\hline$\quad$ Occipital & 23 & 13 & \\
\hline$\quad$ Frontal & 16 & 0 & \\
\hline Subsequent revision w/in 1 mo & $7(18 \%)$ & $2(15 \%)$ & 0.68 \\
\hline Average time until subsequent & & & \\
$\quad$ revision (days) & 47 & 219 & 0.13 \\
\hline Difficult catheter removal & 5 & 8 & $<0.01$ \\
\hline
\end{tabular}

* Values in bold type are statistically significant. 

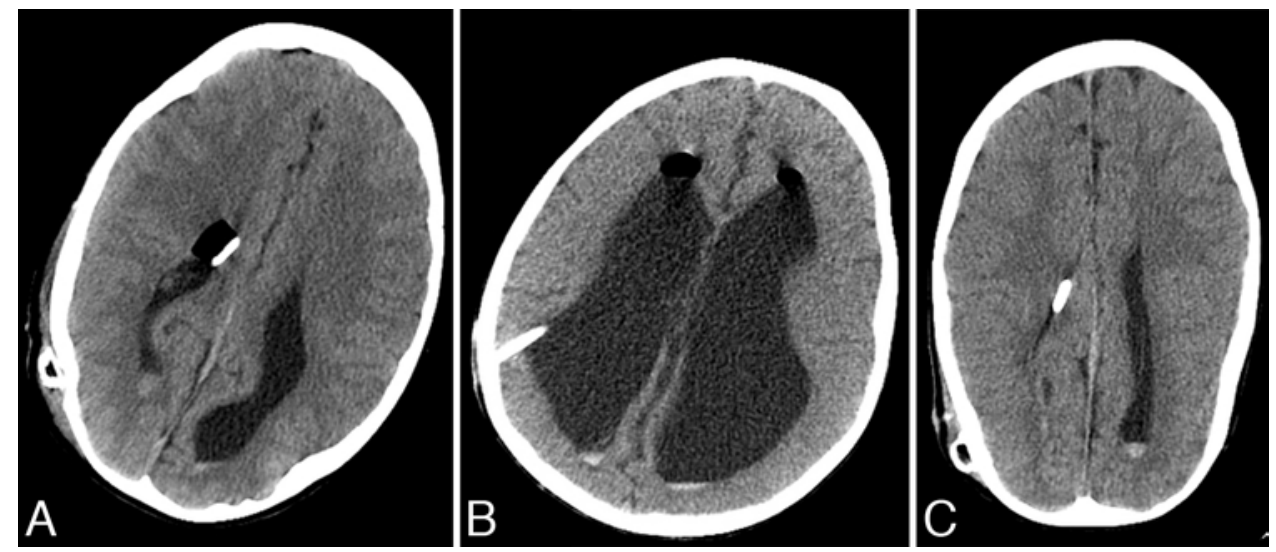

FIG. 1. Representative postoperative axial CT scans obtained in 3 patients who underwent a VP shunt revision with IVH measuring less than $5 \mathrm{ml}$.

prior to our series. Surprisingly, there were some differences between our results.

First, the rate of IVH differed between the 2 series. Brownlee et al. ${ }^{3}$ had an IVH rate of $31 \%$ versus $25 \%$ in our series. However, for IVH greater than $5 \mathrm{ml}$, our rate was $2 \%$. One reason for this discrepancy, besides the difference in the number of patients in each series (247 patients vs 32 patients with 52 revisions in our series), may be the fact that they included bloody CSF encountered after the removal of the catheter as well as IVH found on CT. A good portion of these observations probably represented minor bleeding along the catheter tract rather than true IVH, as IVH was confirmed by CT in only $63 \%$ of the cases in which bleeding was observed. Similarly, our rate of $25 \%$, when taking into account any amount of IVH postoperatively, probably does not represent true IVH from choroidal or ependymal bleeding. The images shown in Fig. 1 are representative of the majority of CT scans demonstrating IVH, with volume less than $5 \mathrm{ml}$, and this is likely due to minor bleeding from the tract settling in the atria. Unfortunately, Brownlee et al. did not report IVH volume in their study.

Second, despite the presence of blood within the ventricle, the average time to the next revision was longer in these cases than for cases in which there was no evidence of IVH (219 days vs 47 days). This was in contrast to the findings of Brownlee et al., ${ }^{3}$ who reported that patients with postoperative IVH had a shorter average time to the next revision (227 days) than patients without IVH (735 days). One reason for the difference in the findings of the 2 studies could be the volume of IVH encountered in the series reported by Brownlee et al. compared with our series, although again they do not mention specifics about the volume of intraventricular blood in their cases. As mentioned earlier, when IVH did occur in our series, the volume was less than $5 \mathrm{ml}$ except in one instance, and in that one exception the patient did not require a subsequent revision (Fig. 2). This could be related to irrigating the ventricle, if there was a suspicion for IVH, until the CSF was clear. Clearing or minimizing the IVH could decrease the chance for early failure. Another reason for the discrepancy between the average time to revision among patients with IVH could be due to our smaller sample size. A larger sample size may show that patients with IVH have a similar or a shorter average time to their next revision.

Knowing the rate of IVH associated with VP shunt revisions and the acute consequences has some practical applications. There is a perception among some neurosurgeons that patients with long-standing ventricular catheters are at increased risk for developing IVH during a proximal revision. Again, this perception is supported by evidence that the presence of the catheter prompts growth of the choroid plexus around it over time, leading to a potentially risky removal. As a consequence, the practice of
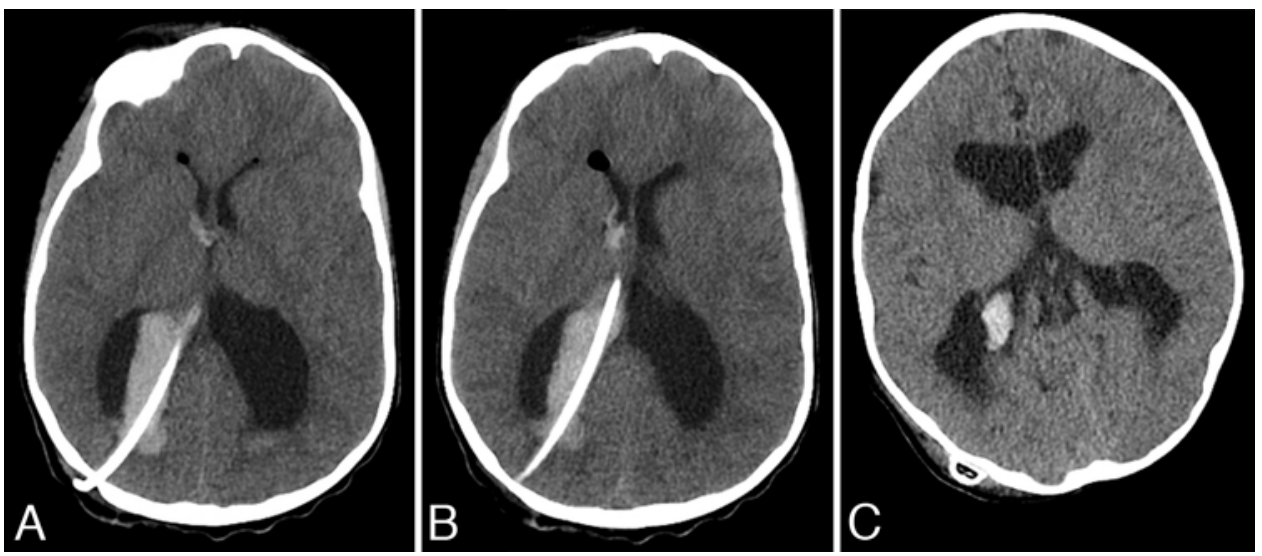

FIG. 2. Axial CT scans demonstrating a postoperative IVH that was greater than $5 \mathrm{ml}$ in one of our patients. 
placing a new ventricular catheter at a new site rather than removing an old existing catheter, particularly if there is reason to believe the catheter is especially adherent, has developed and has been advocated in the literature. ${ }^{2,11}$ This practice can lead to complicated and often confusing shunt systems. Furthermore, revising these systems in the setting of shunt failure or even worse, removing multiple shunts because of infection, can be a daunting task. However, knowing that the chances of major bleeding are low, simply revising the single catheter can prevent complicated revisions in the future. In the adult literature, the risk of any intracranial hemorrhage from a de novo VP shunt is $4 \%$ and the risk of IVH is $2.4 \%$. This suggests that removing a proximal VP shunt catheter is as safe if not slightly safer than placing a new shunt..$^{10}$ In our study there was a correlation between IVH found postoperatively and the difficulty in removing the catheter as documented by our senior author (M.A.A.); however, only 1 patient required an early revision.

Interestingly, we found in our study that the only VP shunt catheter revision procedures that led to IVH were revisions of occipitally placed catheters. Older literature supported placing the proximal catheter either anterior to the foramen of Monro ${ }^{6}$ to avoid the choroid plexus or posteriorly, within the atrium or occipital horns, ${ }^{8,9}$ to prevent obstruction. More recent literature suggests that the ideal placement of a VP shunt catheter should be superior to the foramen of Monro or within the body of the lateral ventricle to prevent occlusion. ${ }^{12}$ Catheters that are placed from an occipital bur hole course along the long axis of the ventricle, thus putting them alongside the course of the choroid plexus. This proximity makes it likely for the catheter to become infiltrated or entangled with the choroid, increasing the risk for IVH upon its removal.

Some limitations to this study include potential selection bias and recall bias because of its retrospective nature. Also, the study lacks sufficient power to show any significant correlation between IVH and the need for revision, because of the relatively low number of patients. A prospective study with a larger number of patients or, ideally, a multicenter study would better elucidate the rates of VP shunt revision and associated complications. Finally, there are cases in which IVH occurs after a revision procedure without any clear cause. A study involving in vivo visualization of the catheter replacement may be beneficial in determining a more accurate rate and the various etiologies of IVH.

\section{Conclusions}

Our study found that the rate of significant IVH due to removal of a ventricular catheter is relatively low, at $2 \%$. When postoperative IVH does occur, our data suggest that patients will remain asymptomatic and will likely not require an immediate revision. This is important in terms of giving surgeons confidence in removing long-standing catheters and avoiding the creation of more complicated shunt systems in their patients.

\section{Acknowledgment}

A special thank you to Dr. Paul Feustel for his invaluable assistance with the statistical analysis of the data in this study.

\section{References}

1. Andersson H, Carlsson CA: Plexectomy as an aid in blocked ventriculo-venous shunts. Neurochirurgia (Stuttg) 9:61-65, 1966

2. Becker DP, Nulsen FE: Control of hydrocephalus by valveregulated venous shunt: avoidance of complications in prolonged shunt maintenance. J Neurosurg 28:215-226, 1968

3. Brownlee RD, Dold ON, Myles ST: Intraventricular hemorrhage complicating ventricular catheter revision: incidence and effect on shunt survival. Pediatr Neurosurg 22:315320, 1995

4. Collins P, Hockley AD, Woollam DHM: Surface ultrastructure of tissues occluding ventricular catheters. J Neurosurg 48:609-613, 1978

5. Forrest DM, Cooper DGW: Complications of ventriculo-atrial shunts. A review of 455 cases. J Neurosurg 29:506-512, 1968

6. Giuffrè R: Choroid and ependymal reactions. J Neurosurg Sci 20:123-129, 1976

7. Kothari RU, Brott T, Broderick JP, Barsan WG, Sauerbeck LR, Zuccarello M, et al: The ABCs of measuring intracerebral hemorrhage volumes. Stroke 27:1304-1305, 1996

8. Sainte-Rose C: Shunt obstruction: a preventable complication? Pediatr Neurosurg 19:156-164, 1993

9. Sainte-Rose C, Piatt JH, Renier D, Pierre-Kahn A, Hirsch JF, Hoffman HJ, et al: Mechanical complications in shunts. Pediatr Neurosurg 17:2-9, 1991-1992

10. Savitz MH, Bobroff LM: Low incidence of delayed intracerebral hemorrhage secondary to ventriculoperitoneal shunt insertion. J Neurosurg 91:32-34, 1999

11. Singh D, Saxena A, Jagetia A, Singh H, Tandon MS, Ganjoo P: Endoscopic observations of blocked ventriculoperitoneal (VP) shunt: a step toward better understanding of shunt obstruction and its removal. Br J Neurosurg 26:747-753, 2012

12. Yamada SM, Kitagawa R, Teramoto A: Relationship of the location of the ventricular catheter tip and function of the ventriculoperitoneal shunt. J Clin Neurosci 20:99-101, 2013

\section{Author Contributions}

Conception and design: all authors. Acquisition of data: Calayag, Paul. Analysis and interpretation of data: all authors. Drafting the article: Calayag. Critically revising the article: Adamo, Calayag. Reviewed submitted version of manuscript: Adamo, Calayag. Statistical analysis: Paul. Study supervision: Adamo, Calayag.

\section{Correspondence}

Matthew A. Adamo, Department of Neurosurgery, Albany Medical Center, 43 New Scotland Ave., MC-10, Albany, NY 12208. email: adamom@mail.amc.edu. 